\title{
A CHARACTERIZATION OF COBOUNDARY POISSON LIE GROUPS AND HOPF ALGEBRAS
}

\author{
STANISŁAW ZAKRZEWSKI \\ Department of Mathematical Methods in Physics, University of Warsaw \\ Hoża 74, 00-682 Warszawa, Poland \\ E-mail:szakrz@fuw.edu.pl
}

\begin{abstract}
We show that a Poisson Lie group $(G, \pi)$ is coboundary if and only if the natural action of $G \times G$ on $M=G$ is a Poisson action for an appropriate Poisson structure on $M$ (the structure turns out to be the well known $\pi_{+}$). We analyze the same condition in the context of Hopf algebras. A quantum analogue of the $\pi_{+}$structure on $S U(N)$ is described in terms of generators and relations as an example.
\end{abstract}

1. Preliminaries. For the theory of Poisson Lie groups we refer to $[1,2,3,4,5]$. We follow the notation used in our previous papers $[6,7]$.

A Poisson Lie group is a Lie group $G$ equipped with a Poisson structure $\pi$ such that the multiplication map is Poisson. The latter property is equivalent to the following property (called multiplicativity of $\pi$ ):

$$
\pi(g h)=\pi(g) h+g \pi(h) \quad \text { for } g, h \in G .
$$

Here $\pi(g) h$ denotes the right translation of $\pi(g)$ by $h$ etc. This notation will be used throughout the paper.

A Poisson Lie group is said to be coboundary if

$$
\pi(g)=r g-g r
$$

for a certain element $r \in \mathfrak{g} \wedge \mathfrak{g}$. Here $\mathfrak{g}$ denotes the Lie algebra of $G$. Any bivector field of the form (2) is multiplicative. It is Poisson if and only if

$$
[r, r] \in(\mathfrak{g} \wedge \mathfrak{g} \wedge \mathfrak{g})_{\text {inv }}
$$

(the Schouten bracket $[r, r]$ is $\mathfrak{g}$-invariant). In this case the element $r$ is said to be a classical r-matrix (on $\mathfrak{g})$.

1991 Mathematics Subject Classification: Primary 17B37, 16W30; Secondary 81S05.

Research supported by KBN grant 2 P301 02007.

The paper is in final form and no version of it will be published elsewhere. 
For any Poisson Lie group $(G, \pi)$, the antipode map $g \mapsto S g:=g^{-1}$ is anti-Poisson:

$$
S_{*} \pi=-\pi \text {. }
$$

2. Gauge transformations of a lattice connection on one link. Consider the following action

$$
(G \times G) \times G \ni\left(\left(g_{0}, g_{1}\right), g\right) \mapsto g_{1} g g_{0}^{-1} \in G
$$

of $G \times G$ on $G$. This type of action is familiar in gauge field theory on the lattice. We think here about an 'elementary' lattice composed of only one link with two ends: 0 and 1. Elements $g_{0}$ and $g_{1}$ are the values of the gauge transformation at the lattice sites 0 and 1, respectively. The connection on the link is represented by the element $g$.

One can ask if it is possible to consider the gauge group to be a Poisson Lie group (or, a quantum group). In this case it is natural to require the action (4) to be a Poisson action (i.e. the map (4) to be a Poisson map).

Definition 1. A Poisson Lie group $(G, \pi)$ is said to be gauge-admissible if there exists a Poisson structure $\rho$ on $G$ such that the map (4) is a Poisson map as a map from $(G, \pi) \times(G, \pi) \times(G, \rho)$ to $(G, \rho)$.

Note that we treat the gauge group differently than the space of connections (even if the latter is parameterized by the group manifold).

Proposition 1. A Poisson Lie group is gauge admissible if and only if it is coboundary.

Proof. Let $(G, \pi)$ be a Poisson Lie group. It is gauge admissible if and only if the map

$$
G \times G \times G \ni(x, y, z) \mapsto x y z^{-1} \in G
$$

is Poisson as a map from $(G, \pi) \times(G, \rho) \times(G, \pi)$ to $(G, \rho)$ or, equivalently (using $(3)$ ), if the map $\Psi: G \times G \times G \rightarrow G$ defined by

$$
\Psi(x, y, z)=x y z
$$

is Poisson as a map from $(G, \pi) \times(G, \rho) \times(G,-\pi)$ to $(G, \rho)$. By a similar reasoning which leads to (1), this is equivalent to

$$
\rho(x y z)=\pi(x) y z+x \rho(y) z-x y \pi(z) \quad \text { for } \quad x, y, z \in G .
$$

We have two following particular cases of this equality. If we set $z=e$ (the group unit), we get

$$
\rho(x y)=\pi(x) y+x \rho(y)
$$

and if we set $x=e$, we get

$$
\rho(y z)=\rho(y) z-y \pi(z) .
$$

It is easy to see that (7) and (8) together are equivalent to (6). Since $\rho=\pi$ is a particular solution of (7), the general solution of (7) is given by

$$
\rho(g)=\pi(g)+g A,
$$


where $A \in \mathfrak{g} \wedge \mathfrak{g}$. Since $\rho=-\pi$ is a particular solution of (8), the general solution of (8) is given by

$$
\rho(g)=-\pi(g)+B g
$$

where $B \in \mathfrak{g} \wedge \mathfrak{g}$. For the compatibility of (9) and (10) we must have

$$
\pi(g)=\frac{B g-g A}{2} .
$$

Since $\pi(e)=0$, we have $B=A$, and finally

$$
\pi(g)=\frac{A g-g A}{2}, \quad \rho(g)=\frac{A g+g A}{2} .
$$

This shows that $(G, \pi)$ is gauge-admissible if and only if it is coboundary (with $r=A / 2$; note that if $r$ is a classical $r$-matrix then $\pi_{+}(g):=r g+g r=\rho(g)$ is automatically a Poisson bivector field).

It is clear that for a given coboundary Poisson structure $\pi$, all possible $\rho$ are obtained from one by adding an invariant element of $\mathfrak{g} \wedge \mathfrak{g}$. In particular, if $\mathfrak{g}$ is semisimple, then $\rho$ is unique.

3. Hopf algebra case. Let $(H, m, \Delta)$ be a Hopf algebra. Here $m: H \otimes H \rightarrow H$ and $\Delta: H \rightarrow H \otimes H$ denote the multiplication and the comultiplication in $H$. Let $I$ and $c$ denote the unit and counit of the Hopf algebra.

We set

$$
\Psi:=m(m \otimes \mathrm{id})=m(\mathrm{id} \otimes m)
$$

and ask when there exists a (new) coalgebra structure $\widetilde{\Delta}$ (with the same counit $c$ ) on $H$ such that $\Psi$ is a morphism from $(H, \Delta) \otimes(H, \widetilde{\Delta}) \otimes\left(H, \Delta^{\mathrm{op}}\right)$ to $(H, \widetilde{\Delta})$. Here $\Delta^{\mathrm{op}}$ is the comultiplication opposite to $\Delta: \Delta^{\mathrm{op}}=P \circ \Delta$, where $P$ is the permutation in the tensor product.

The condition for $\Psi$ to be such a morphism reads:

$$
\widetilde{\Delta} \Psi=(\Psi \otimes \Psi)(\text { id } \otimes \text { id } \otimes P \otimes \text { id } \otimes \text { id })(\text { id } \otimes P \otimes P \otimes \text { id })\left(\Delta \otimes \widetilde{\Delta} \otimes \Delta^{\mathrm{op}}\right),
$$

and is equivalent to the following two conditions:

$$
\begin{gathered}
\widetilde{\Delta} m=(m \otimes m)(\text { id } \otimes P \otimes \text { id })(\Delta \otimes \widetilde{\Delta}), \\
\widetilde{\Delta} m=(m \otimes m)(\text { id } \otimes P \otimes \text { id })\left(\widetilde{\Delta} \otimes \Delta^{\mathrm{op}}\right)
\end{gathered}
$$

(they follow from (11) by applying it to id $\otimes$ id $\otimes I$ and $I \otimes$ id $\otimes$ id, respectively). It is easy to solve these conditions for $\widetilde{\Delta}$. Applying (12) to id $\otimes I$, we get

$$
\widetilde{\Delta}(a)=\Delta(a) \mathbf{R} \quad a \in H,
$$

where the multiplication is that of $H \otimes H$ and

$$
\mathbf{R}:=\widetilde{\Delta}(I) .
$$

It is easy to see that (14) solves (12) for any $\mathbf{R}$.

Similarly, applying (13) to $I \otimes$ id, we get

$$
\widetilde{\Delta}(a)=\mathbf{R} \Delta^{\mathrm{op}}(a) \quad a \in H .
$$


This is a solution of (13) for any $\mathbf{R}$. It follows that the general solution of (11) is (14), where the $R$-matrix $\mathbf{R}$ satisfies the compatibility condition

$$
\Delta(a) \mathbf{R}=\mathbf{R} \Delta^{\mathrm{op}}(a) \quad a \in H .
$$

It is easy to see that $\widetilde{\Delta}$ is coassociative if and only if

$$
[(\Delta \otimes \mathrm{id}) \mathbf{R}](\mathbf{R} \otimes I)=[(\mathrm{id} \otimes \Delta) \mathbf{R}](I \otimes \mathbf{R}) .
$$

Indeed,

$$
\begin{aligned}
(\widetilde{\Delta} \otimes \mathrm{id}) \widetilde{\Delta}(a) & =[(\Delta \otimes \mathrm{id})(\Delta(a) \mathbf{R})](\mathbf{R} \otimes \mathrm{id})=[(\Delta \otimes \mathrm{id}) \Delta(a)][(\Delta \otimes \mathrm{id}) \mathbf{R}](\mathbf{R} \otimes \mathrm{id}), \\
(\mathrm{id} \otimes \widetilde{\Delta}) \widetilde{\Delta}(a) & =[(\mathrm{id} \otimes \Delta)(\Delta(a) \mathbf{R})](\mathrm{id} \otimes \mathbf{R})=[(\mathrm{id} \otimes \Delta) \Delta(a)][(\mathrm{id} \otimes \Delta) \mathbf{R}](\mathrm{id} \otimes \mathbf{R}) .
\end{aligned}
$$

Concluding: the question at the beginning of this section has an affirmative answer if and only if there exists an element $\mathbf{R} \in H \otimes H$ such that (15), (16) hold and

$$
(c \otimes \text { id }) \mathbf{R}=I=(\text { id } \otimes c) \mathbf{R} .
$$

A Hopf algebra satisfying those conditions might be called gauge-admissible or coboundary.

Notice that the definition of a coboundary Hopf algebra originally introduced by Drinfeld [2] requires also the 'unitarity' of $\mathbf{R}$, i.e. $\mathbf{R}_{12} \mathbf{R}_{21}=I \otimes I$. However, this condition does not seem to be really a restriction. If it is not satisfied, one can use the Drinfeld's prescription:

$$
\overline{\mathbf{R}}:=\left(\mathbf{R}_{12} \mathbf{R}_{21}\right)^{-\frac{1}{2}} \mathbf{R}
$$

(assuming the existence of the square root with suitable properties; for example, one can assume the situation of a QUE-algebra) to pass to another element which is already 'unitary' and satisfies (15), (16).

The Hopf algebra considered in this section should be interpreted as a dual of the Hopf algebra of functions on a quantum group (quantized universal enveloping algebra). In the next section we give an example of a 'gauge-admissible' matrix quantum group.

\section{Example in terms of generators and relations. Let}

$$
R(u \oplus u)=(u \text { (1) } R
$$

be a part of relations defining a matrix quantum group $(A, u)$. Here $u=\left(u_{i j}\right)_{i, j=1, \ldots, n}$ is the defining representation of the quantum group, $R$ is the fundamental intertwiner ( $R$-matrix of FRT-type) and we use the Woronowicz's notation for the 'matrix' tensor product. Let us note that we have

$$
\widetilde{R}\left(u^{-1} \text { (1) } u^{-1}\right)=\left(u^{-1} \text { (1) } u^{-1}\right) \widetilde{R}
$$

where $\widetilde{R}:=P R P$. Let us denote by $B$ the algebra generated by the entries of the $n \times n$ matrix $w$ and relations

$$
R(w \oplus w)=(w \oplus w) \widetilde{R}
$$

It is easy to see that there exists exactly one homomorphism $\pitchfork$ (quantum gauge transformation - the analogue of (5)) from $B$ to $A \otimes B \otimes A$ such that

$$
\pitchfork\left(w_{j}^{i}\right)=\sum_{k l} u_{k}^{i} \otimes w^{k}{ }_{l} \otimes\left(u^{-1}\right)^{l}{ }_{j}
$$


or, using Woronowicz's notation,

$$
(\pitchfork \otimes \mathrm{id})(w)=u(1) w \oplus u^{-1}
$$

(here $w$ is understood as an element of $\operatorname{End}\left(\mathbb{C}^{n}\right) \otimes B$ ). In order to see that $u \oplus w \oplus u^{-1}$ satisfies the same relations as $w$, we notice that

$$
\left(u \oplus w \oplus u^{-1}\right) \oplus\left(u \oplus w \oplus u^{-1}\right)=(u(1)) \oplus(w \oplus w) \oplus\left(u^{-1} \oplus u^{-1}\right)
$$

and use subsequently (18), (19) and (20).

In order to be more precise, we consider now a specific matrix quantum group, namely $S U_{q}(n)$, as given in [8]. The *-algebra $A$ of 'regular functions' on $S U_{q}(n)$ is the one generated by the entries of an $n \times n$ matrix $u$ and the following relations:

$$
u^{(n)} E=E, \quad E^{\prime} u^{(n)}=E^{\prime}, \quad u u^{*}=I_{n} \otimes I_{A}=u^{*} u .
$$

Here $u^{(n)}$ is the $n$-th tensor power of $u, E$ is the ' $q$-deformed' volume element

$$
E^{i_{1} i_{2} \ldots i_{n}}=(-q)^{\text {number of inversions in }\left(i_{1}, \ldots, i_{n}\right)}, \quad \quad E_{i_{1} \ldots i_{n}}^{\prime}=E^{i_{1} \ldots i_{n}}
$$

(for $\left(i_{1} \ldots i_{n}\right)$ not being a permutation we set $E^{i_{1} \ldots i_{n}}=0$ ) and $I_{n}$ is the unit $n \times n$ matrix. Note that in this case

$$
\left(u^{-1}\right)^{(n)} \widetilde{E}=t E, \quad \widetilde{E}^{\prime}\left(u^{-1}\right)^{(n)}=\widetilde{E}^{\prime}
$$

where

$$
\widetilde{E}=P_{\text {total }} E, \quad \widetilde{E}^{\prime}=E^{\prime} P_{\text {total }},
$$

$P_{\text {total }}$ being the total permutation $(1,2, \ldots, n) \mapsto(n, \ldots, 2,1)$.

Let $B$ be the *-algebra generated by the entries of an $n \times n$ matrix $w$ and relations (22) $\quad w^{(n)} \widetilde{E}=(-1)^{\frac{n(n-1)}{2}} E, \quad E^{\prime} w^{(n)}=(-1)^{\frac{n(n-1)}{2}} \widetilde{E}^{\prime}, \quad w w^{*}=I_{n} \otimes I_{B}=w^{*} w$.

It is easy to check that $u(1) w(1) u^{-1}$ satisfies the same relations, hence we have the 'gauge transformations' on the quantum level.

It is essential to know if algebra $B$ has a correct size (Poincaré series), i.e. if the deformation is flat. We shall show that $B$ is actually isomorphic to $A$. To this end, consider the change of variables

$$
u=\varepsilon w P_{\text {total }}
$$

in (21), where $\varepsilon$ is a complex number such that $\varepsilon^{n}=(-1)^{\frac{n(n-1)}{2}}=\operatorname{det} P_{\text {total }}$. It is easy to see that relations (21) are now transformed to relations (22).

\section{Remarks}

5.1. The algebra $B$ defined in (22) is the quantum counterpart of the Poisson structure $\pi_{+}(g)=r g+g r$ on $S U(n)$. The case of a general group is sketched in (20). Note that if we substitute $u=w g_{0}$ in (18) where $g_{0}$ is an element of the classical group such that

$$
\left(g_{0} \otimes g_{0}\right) R\left(g_{0}^{-1} \otimes g_{0}^{-1}\right)=P R P,
$$

then we obtain relations (20). One can check that the well known $R$-matrix for the $A_{n}$ series satisfies (23) if we choose $g_{0}=\varepsilon P_{\text {total }}$. The corresponding fact for Poisson groups means that we find an element $g_{0} \in G$ such that

$$
\pi=\pi_{+} g_{0}
$$


i.e. $\pi\left(g g_{0}\right)=\pi_{+}(g) g_{0}$, that is to say

$$
r g g_{0}-g g_{0} r=r g g_{0}+g r g_{0}
$$

or,

$$
g_{0} r g_{0}^{-1}=-r
$$

or,

$$
\pi_{+}\left(g_{0}\right)=0 .
$$

For instance in the case of the standard $r$-matrix of the $A_{n}$-series,

$$
r=\sum_{j<k} e_{j}^{k} \wedge e_{k}^{j}
$$

$g_{0}:=\varepsilon P_{\text {total }}$ will do the job, because $P e_{j}=e_{j^{\prime}}, j^{\prime}:=n+1-j$.

5.2. Formula (14) was used in [9] to discuss twisting Hopf algebras by 2-cocycles. The Poisson structure $\pi_{+}$is isomorphic to $\pi$ by a translation (24) if and only if it vanishes at some point (namely $g_{0}$, see (25)). This situation (and previously discussed isomorphism of $B$ with $A$ ) corresponds to twisting by a coboundary.

5.3. The author would like to thank Jiang-Hua Lu, Shahn Majid and Marco Tarlini for enlightening discussions.

The author is also very much indebted to Prof. V. Drinfeld for presenting the proof of the fact that (17) satisfies (16), if $\mathbf{R}$ satisfies (15) and (16).

\section{References}

[1] V. G. Drinfeld, Hamiltonian structures on Lie groups, Lie bialgebras and the meaning of the classical Yang-Baxter equations, Soviet Math. Dokl. 27 (1983), 68-71.

[2] V. G. Drinfeld, Quantum groups, Proc. ICM, Berkeley, 1986, vol.1, 789-820.

[3] M. A. Semenov-Tian-Shansky, Dressing transformations and Poisson Lie group actions, Publ. Res. Inst. Math. Sci., Kyoto University 21 (1985), 1237-1260.

[4] J.-H. Lu and A. Weinstein, Poisson Lie Groups, Dressing Transformations and Bruhat Decompositions, J. Diff. Geom. 31 (1990), 501-526.

[5] J.-H. Lu, Multiplicative and affine Poisson structures on Lie groups, Ph.D. Thesis, University of California, Berkeley (1990).

[6] S. Zakrzewski, Poisson structures on the Lorentz group, Lett. Math. Phys. 32 (1994), 11-23.

[7] S. Zakrzewski, Poisson homogeneous spaces, in: "Quantum Groups, Formalism and Applications", Proceedings of the XXX Winter School on Theoretical Physics 14-26 February 1994, Karpacz, J. Lukierski, Z. Popowicz, J. Sobczyk (eds.), Polish Scientific Publishers PWN, Warsaw 1995, pp. 629-639.

[8] S. L. Woronowicz, Tannaka-Krein duality for compact matrix pseudogroups. Twisted $S U(N)$ groups, Invent. Math. 93 (1988), 35.

[9] J.-H. Lu, On the Drinfeld double and the Heisenberg double of a Hopf algebra, Duke Math. Journ. 74, No.3 (1994), 763-776. 